\title{
Feasibility and impact study of a reward-based mobile application to improve adolescents' snacking habits
}

\author{
Nathalie De Cock ${ }^{1} *$, Wendy Van Lippevelde ${ }^{2}$, Jolien Vangeel ${ }^{3}$, Melissa Notebaert $^{3}$, \\ Kathleen Beullens ${ }^{3}$, Steven Eggermont ${ }^{3}$, Benedicte Deforche ${ }^{2,4}$, Lea Maes ${ }^{2}$, \\ Lien Goossens ${ }^{5}$, Sandra Verbeken ${ }^{5}$, Ellen Moens ${ }^{5}$, Leentje Vervoort ${ }^{5}$, Caroline Braet ${ }^{5}$, \\ Lieven Huybregts ${ }^{6}$, Patrick Kolsteren ${ }^{1}$, John Van Camp ${ }^{1}$ and Carl Lachat ${ }^{1}$ \\ 'Department of Food Safety and Food Quality, Ghent University, Coupure Links 653, Ghent 9000, Belgium: \\ ${ }^{2}$ Department of Public Health, Ghent University, Ghent, Belgium: ${ }^{3}$ Leuven School for Mass Communication Research, \\ KU Leuven, Leuven, Belgium: ${ }^{4}$ Physical Activity, Nutrition and Health Research Unit, Faculty of Physical Education and \\ Physical Therapy, Vrije Universiteit Brussel, Brussels, Belgium: ${ }^{5}$ Department of Developmental, Personality and Social \\ Psychology, Ghent University, Ghent, Belgium: 'Poverty, Health and Nutrition Division, International Food Policy \\ Research Institute, Washington DC, USA
}

Submitted 12 October 2017: Final revision received 12 February 2018: Accepted 22 February 2018: First published online 2 April 2018

\begin{abstract}
Objective: Adolescents' snacking habits are driven by both explicit reflective and implicit hedonic processes. Hedonic pathways and differences in sensitivity to food rewards in addition to reflective determinants should be considered. The present study evaluated the feasibility and impact of a mobile phone-delivered intervention, incorporating explicit reflective and implicit rewarding strategies, on adolescents' snack intake.

Design: Adolescents ( $n$ 988; mean age 14.9 (SD 0.70) years, 59.4\% boys) completed a non-randomized clustered controlled trial. Adolescents ( $n$ 416) in the intervention schools ( $n 3$ ) were provided with the intervention application for four weeks, while adolescents ( $n$ 572) in the control schools ( $n$ 3) followed the regular curriculum. Outcomes were differences in healthy snacking ratio and key determinants (awareness, intention, attitude, self-efficacy, habits and knowledge). Process evaluation data were collected via questionnaires and through log data of the app.

Results: No significant positive intervention effects on the healthy snack ratio $(b=-3.52$ (sE 1.82), $P>0.05$ ) or targeted determinants were observed. Only 268 adolescents started using the app, of whom only fifty-five (20.5\%) still logged in after four weeks. Within the group of users, higher exposure to the app was not significantly associated with positive intervention effects. App satisfaction ratings were low in both high and low user groups. Moderation analyses revealed small positive intervention effects on the healthy snack ratio in high compared with low reward-sensitive boys ( $b=1.38$ (se 0.59 ), $P<0 \cdot 05$ ).

Conclusions: The intervention was not able to improve adolescents' snack choices, due to low reach and exposure. Future interventions should consider multicomponent interventions, teacher engagement, exhaustive participatory app content development and tailoring.
\end{abstract}

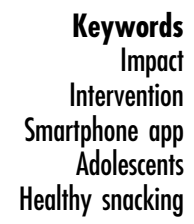

Adolescence is a crucial period for the adoption of eating habits $^{(1,2)}$. Dietary patterns that develop during adolescence track into adulthood and have implications for the development of chronic diseases later in life ${ }^{(3,4)}$. Adolescents have increased energy and nutrient requirements to account for growth and physiological, psychosocial and cognitive development ${ }^{(1,2)}$. The overconsumption of energy-dense and nutrient-poor snack foods, such as candy or chocolate bars, between meals ${ }^{(5-7)}$ and the associated excess energy, sugar and fat intakes among adolescents ${ }^{(3,5,8)}$, however, are of great concern. On the other hand, healthy snacking could help meet the recommendations for essential food groups such as fruit and dairy ${ }^{(5-7)}$. The promotion of healthier snacking behaviour in adolescents is thus warranted.

Most theory-based interventions to improve the dietary behaviours of adolescents have focused on changing psychosocial determinants ${ }^{(9,10)}$. Eating behaviour, however, is the result of the joint function between explicit 
(reflective/psychosocial) cognitive efforts to build beliefs and implicit (habitual/automatic) processes, linkages of certain stimuli or cues to certain behaviour based on earlier learned associations ${ }^{(11-13)}$. Key determinants of the reflective system are, for instance, attitude and self-efficacy; for the implicit system, on the other hand, these are habits ${ }^{(14)}$. The implicit or habitual nature of eating ${ }^{(14-16)}$, and more specifically of snacking in adolescents ${ }^{(7)}$, was only recently recognized. Effective strategies to influence the explicit processes can be derived from the meta-analysis by Michie et $a l^{(17)}$ : interventions combining self-monitoring with at least one other technique derived from the control theory of Carver and Scheier ${ }^{(18)}$ (such as goal setting or providing feedback) were the most effective to improve eating or physical activity behaviours. As habitual snacking might be driven by the higher reinforcing value of energy-dense snacks compared with healthy snacks such as fruits and vegetables $^{(19-25)}$, positive reinforcement might be a good strategy to implicitly increase healthy snack intake. Offering rewards has already been shown to increase the reinforcing value and the consumption of healthy foods in children and adolescents $^{(26-29)}$.

Personal characteristics have shown to determine how individuals react to different behaviour change strategies in children and adolescents ${ }^{(7,26,30,31)}$. Personality theories assume that unique individual characteristics play a role in the expression of eating behaviour ${ }^{(32,33)}$. Sensitivity to reward (SR) is a psychobiological trait, which can be defined as the tendency to engage in motivated approach behaviour in the presence of rewarding stimuli ${ }^{(32,34,35)}$. Individual differences in SR were associated with adolescents' snack intake ${ }^{(36)}$. Rewarding strategies were already found to work better in high SR $v$. low SR toddlers in improving willingness to taste ${ }^{(26)}$. Following the definition of SR, it would thus be expected that rewarding strategies might work better in high SR adolescents in promoting healthy snack intake. However, the relationship between SR and adolescents' snack intake was found to be moderated by sex ${ }^{(26,36)}$. In addition, differences in SR between boys and girls exist ${ }^{(26,33,35)}$. When evaluating the effect of rewarding strategies in improving adolescents' snack intakes, moderation by sex and SR should therefore be considered.

Eighty-six per cent of the adolescents in Flanders own a mobile phone and have on average ten to twenty mobile applications (apps) installed on the device ${ }^{(37)}$; thus, an app might be an interesting delivery platform for health interventions in adolescents. Furthermore, apps provide engaging and affordable ways to promote healthy lifestyle behaviours in adolescents ${ }^{(38-40)}$. Recent mobile health (mHealth) interventions to change adults', adolescents' or children's health behaviours have already produced some promising findings, however with modest effect sizes $^{(38,41,42)}$. In addition, few studies report on the feasibility, the acceptability of the intervention and/or provide user statistics for the app ${ }^{(42,44)}$. Process evaluation is important in understanding intervention effectiveness, especially in programmes of increasing complexity such as mHealth interventions ${ }^{(44,45)}$. When programmes get more complex, many factors can contribute to unexpected null findings or explain found positive/negative effects ${ }^{(44,45)}$. Process evaluation can give insights into which possible underlying factors might explain why a programme succeeds or fails in effecting change ${ }^{(44,45)}$.

The present study evaluated both the feasibility (process evaluation) and impact of the 'Snack Track School' app intervention in adolescents. Positive effects were expected on adolescents' healthy snack intakes and targeted determinants. The intervention encompassed both rewarding strategies to influence the implicit/automatic processes and reflective methods derived from the control theory to target the explicit pathways. In addition, moderation of the intervention effects by SR and sex was assessed.

\section{Methods}

The present research forms the concluding study of the REWARD project's adolescent work package ${ }^{(46)}$. REWARD (2013-2016) was a multidisciplinary project that aimed to research and improve the nutritional status of children and adolescents by focusing on SR, rewarding paradigms and learning theory.

\section{Overview and design}

The study design entailed a four-week pre-post controlled clustered trial conducted from January until April 2016 in six secondary schools (three intervention schools, three control schools) in two (matched) cities with comparable socio-economic characteristics, population density and size in Flanders, Belgium. A controlled cluster trial was chosen over a (cluster) randomized controlled trial because of practical and budgetary considerations. In addition, the REWARD intervention included a participatory app development approach, which required long-term engagement and support of the local government, school principals and teachers. The teachers and principals were involved in the app development for two years and wanted then to host the intervention in their schools. To minimize differences between adolescents in the intervention and the control group, however, control schools were selected from a city with comparable socio-economic characteristics, population density and size.

The adolescents in the intervention schools received a four-week mobile app intervention, called 'Snack Track School'. The control schools continued their usual school curriculum and practices. The full study period consisted of a pre-test, the four-week intervention and a post-test immediately after the intervention. Approval for the trial was provided by the Ethics Committee of the University Hospital of Ghent University and the University of Leuven. 
Consent was obtained from the school authorities (school board and headmasters) and the parents (passive informed consents). The trial was registered at clinicaltrials.gov (number NCT02622165). A full description of the protocol of the intervention study can be found elsewhere $^{(47)}$. Findings are reported following the CONSORT (Consolidated Standards of Reporting Trials) and TREND (Transparent Reporting of Evaluations with Nonrandomized Designs) guidelines ${ }^{(48,49)}$

\section{Participants, sampling, allocation and blinding}

The target population consisted of 14-16-year-old Flemish adolescents (i.e. grade 3 and 4 of Belgian secondary schools). The sample size was calculated based on the healthy snacking ratio, in a three-level cluster design ${ }^{(50)}$. To detect a difference of $20 \%$ between intervention and control at the $5 \%$ significance level with a power of $80 \%$, assuming an intraclass correlation of 0.02 at school and 0.03 at class level, mean and standard deviation of the healthy snacking ratio of $37 \cdot 8$ (SD 20.2) and $33 \%$ oversampling to account for attrition, 1436 adolescents (control and intervention) were needed. The intraclass correlation, mean and standard deviation of the healthy snacking ratio were based on the earlier REWARD studies ${ }^{(36,51)}$. No random allocation of schools, classes or students took place, nor were there any exclusion criteria applied.

\section{Procedure}

The baseline assessment took place in January 2016; adolescents were given two class hours $( \pm 100 \mathrm{~min})$ on a pre-agreed date to complete the survey at school in the presence of the research staff. In this way adolescents could ask for clarification in case some of the questions in the survey were not clear.

The app was launched at the schools in February 2016. Smartphones were provided to adolescents without smartphone, enabling participation of all adolescents. During the launch of the app, a tutorial on how to download the game and a short introduction stating the main purpose of the app (tracking their snack intake) was given. A tutorial summarizing how to use the app was incorporated in the app. In the first four minutes of the app adolescents were informed about the main app features by one of the app's characters. During the four weeks of the intervention, however, the adolescents received only minimal guidance. Teachers and other school personnel did not provide any additional messages. Researchers visited the intervention schools weekly during the intervention period to solve any arisen problems and to collect feedback about the intervention from the adolescents (focus group discussions, results not presented/ used here).

The post-survey took place in March and April 2016; adolescents were again given two class hours $( \pm 100 \mathrm{~min})$ at school on a pre-agreed date to complete the survey at school in the presence of the research staff.
The CONSORT flowchart detailing the sampled adolescents and the procedure followed is shown in Fig. 1.

\section{Intervention}

\section{Intervention development}

Briefly, the intervention was developed according to the systematic, stepwise, iterative and collaborative principles of the Intervention Mapping protocol ${ }^{(52)}$ and also made use of strong participatory methods. The dual process model $^{(11-13)}$ was used as theoretical framework to describe the theory of change for the intervention, because it consists of both explicit and implicit pathways and allows the inclusion of other theoretical models like rewarding learning models and control theory. A detailed description of the intervention development, theoretical framework, targeted determinants, used behaviour change techniques and the participatory process is documented elsewhere $^{(47)}$. Figure 2, however, provides a short overview of the theoretical basis of the intervention.

\section{'Snack Track School' app}

The app presented a virtual high-school environment with typical school locations such as classrooms and a gym hall. The core elements of the app were a personal snack track tool, a credit and bonus system, a goal-setting booklet and a report card.

The snack track tool allowed the adolescents to register and monitor their individual snack intake. Adolescents could search and select their snack in a large snack database. For instance, if they consumed chocolate, they could search the database for chocolate or the specific brand of chocolate they consumed and then select this. Adolescents were just to complete their snack choice, not the consumed portion. The snack database was constructed based on the Belgian Internubel Trade Name database ${ }^{(53)}$ and contained over 3000 snack foods. For each snack consumed, the adolescents were then awarded credits reflecting its nutrition value.

The credit or points system of the app awarded points according to the UK Ofcom Nutrient Profiling Model $(\mathrm{NPM})^{(54)}$. Points awarded ranged from 0 to 55 , with 0 being very unhealthy and 55 very healthy. The points that adolescents collected during the week contributed to the total amount of points of the group that they were assigned to for that week's challenge, a group competition or cooperation assignment (e.g. boys against girls or the entire group of adolescents of one intervention school working together to keep the virtual school from closing). The bonus system was added to the app to stimulate a balanced snacking pattern and not merely the tracking of as many snacks as possible. Bonuses were awarded according to three gratuities and one limitation was also built into the app. Participants could track as many snacks as they wanted, however they could only earn credits for the first ten snacks. Only ten snacks were allowed because we anticipated three to five snack moments and one or 


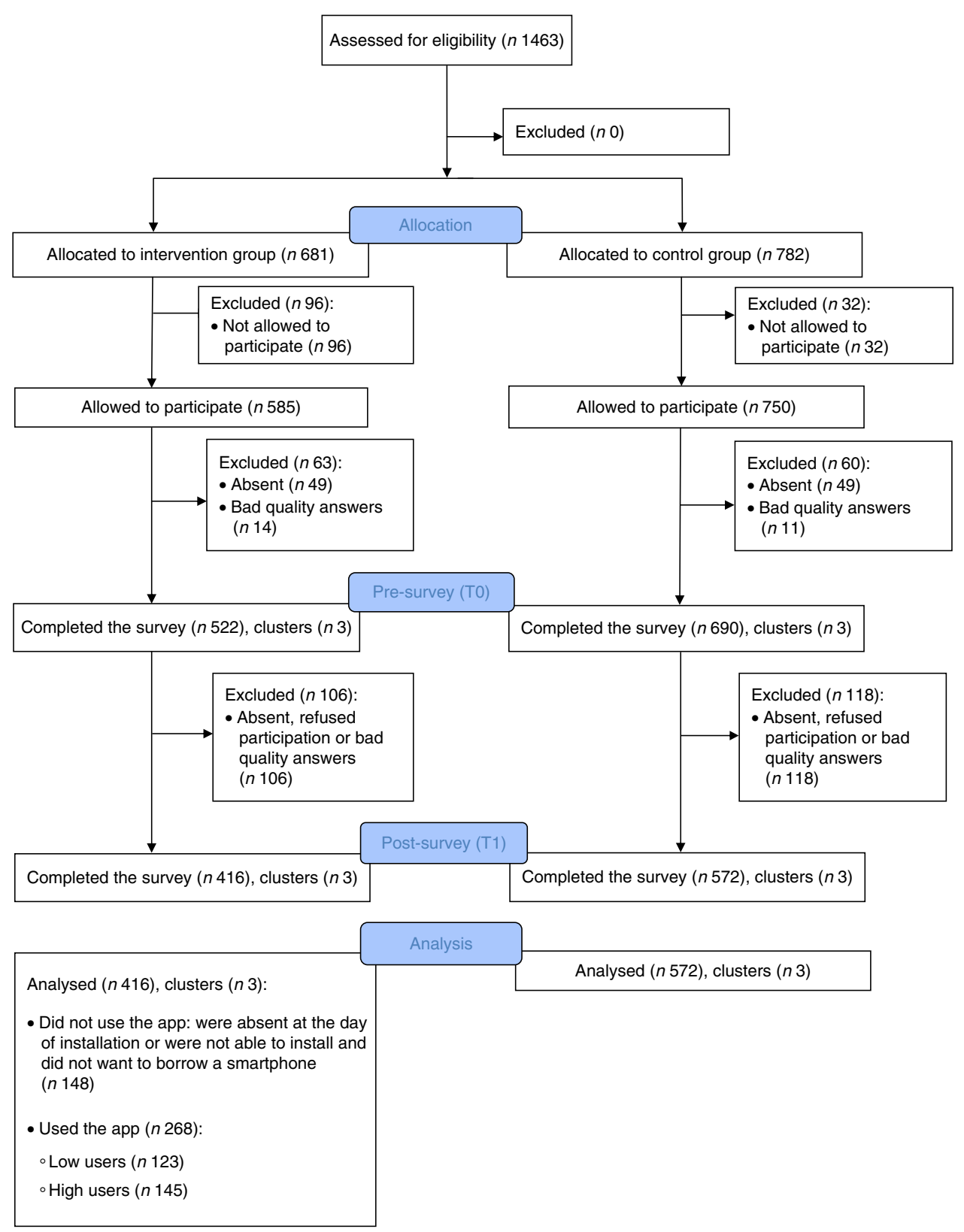

Fig. 1 (colour online) Flowchart of the 'Snack Track School' app intervention

two snacks per snack moment. Recent research on snacking in adolescents in Europe also shows that adolescents eat a snack on average 2-3 times/d, with a maximum of $9-10$ snacks/ $\mathrm{d}^{(55,56)}$. The three gratuities were based on the Flemish guidelines of recommended food and nutrient intakes for adolescents ${ }^{(57)}$, and the full explanation of how these gratuities were developed is given elsewhere $^{(47)}$. Briefly, bonuses of 150 points were given for: (i) a snack intake of $\leq 6$ snacks daily; (ii) a snack intake of $\geq 2 / 3$ healthy snacks out of the total daily snacks; and (iii) not snacking, but involved in the app (logging into the app $\geq 3$ times/d). Additionally, a bonus of 150 points was also given if the participants reached their daily goal.
A goal-setting feature in the form of a booklet was also incorporated in the app. Goal setting was applied from week 2 of the intervention until week 4 . At the beginning of each week participants needed to select one of the four provided goal options, which they then needed to reach every day. In the case of success, the bonus of 150 points was awarded at the end of the day.

At the end of every week, participants also received feedback via a weekly report. This report portrayed all their consumed snacks per day, total credits, credits per snack and the awarded bonuses.

A summary of the different app intervention components and the corresponding behaviour change techniques is given in Table 1 , while screenshots of the 


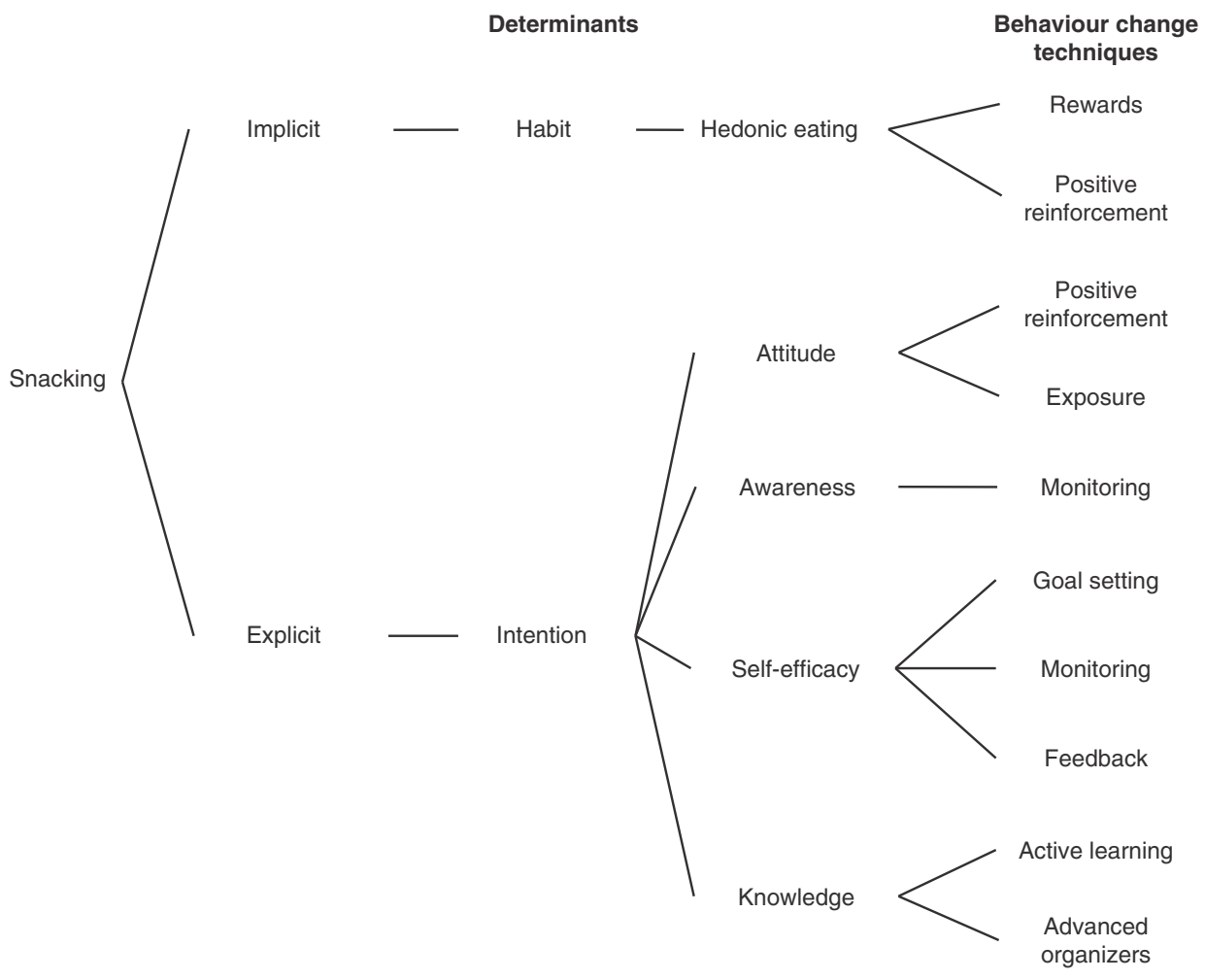

Fig. 2 Overview of the targeted determinants and their corresponding behaviour change techniques

Table 1 Overview of the intervention components used in the 'Snack Track School' app

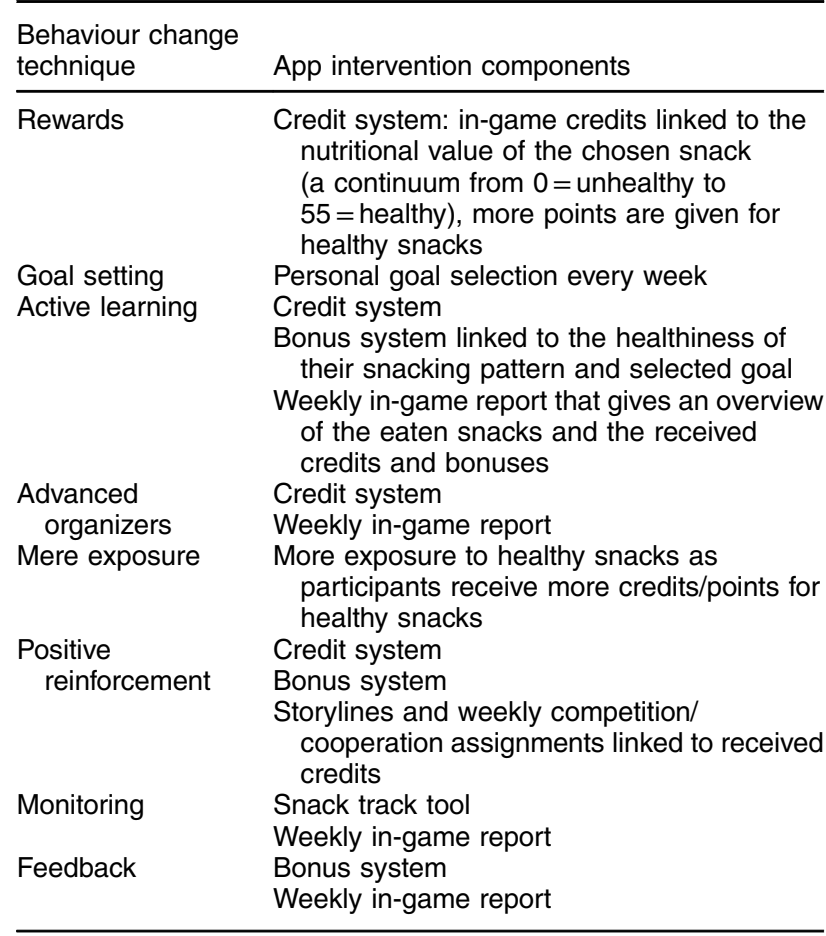

intervention components, the snack track tool, the credit system, the goal-setting booklet and the report card are shown in Fig. 3.
To increase adolescents' feelings of engagement and gamification, several game features were also included. Every week had its own storyline and challenges embedded in a 'game' environment. Adolescents progressed through these weekly challenges (competition or cooperation group challenges) by their earned points. In addition, a customizable avatar and small assignments were incorporated. The rationale for including these specific game features is explained elsewhere ${ }^{(47)}$.

\section{Measures}

\section{Outcome measures}

Primary outcome. Snack intake was assessed using a validated quantitative snack and beverage FFQ, developed within the REWARD project, that probes for usual snack intake with a reference period of one month ${ }^{(51)}$. The intake of snacks was evaluated in terms of all food items consumed outside (>30 min) breakfast, lunch and dinner ${ }^{(8)}$. Snacks were classified as either unhealthy or healthy using the UK Ofcom NPM, which provides a score that represents the (un)healthiness of a beverage or food product ${ }^{54)}$. The classification of the snacks as healthy or unhealthy can be found in the paper describing the validation of the FFQ ${ }^{(51)}$. For each FFQ category, the usual daily intake was calculated by multiplying the frequency of consumption with the quantity of consumption per week (grams) divided by 7 . These daily intakes were then summed to obtain the daily intake of healthy snacks (grams) and unhealthy snacks 
(grams). Subsequently a healthy snack ratio was also calculated. The higher this ratio, the healthier was considered the snack intake of the adolescents:

Healthy snack ratio $=$

$$
\frac{\text { Daily intake of healthy snacks }(\mathrm{g})}{\text { Daily intake healthy and unhealthy snacks }(\mathrm{g})} \times 100 \text {. }
$$

Secondary outcomes. Next to the primary outcomes, secondary effects of the intervention are to be expected on the targeted determinants. The assessment of the constructs awareness, intention, attitude and self-efficacy was based on the reliable and valid healthy diet determinants of the Healthy Lifestyle in Europe by Nutrition in Adolescence (HELENA) study ${ }^{(58)}$. Habit was measured with the automaticity subscale (the 'Self-Report Behavioural Automaticity Index ${ }^{(59)}$ ) of the Self-Report Habit Index ${ }^{(60)}$. More information on these scales can be found in the paper describing

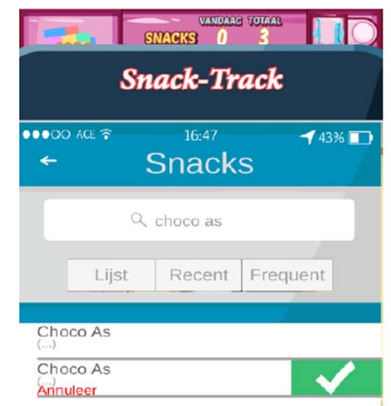

Snack track tool

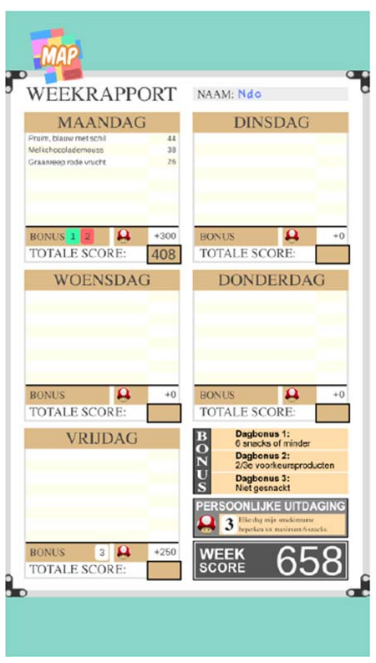

Report card with feedback achievement of chosen goal on bonus system and

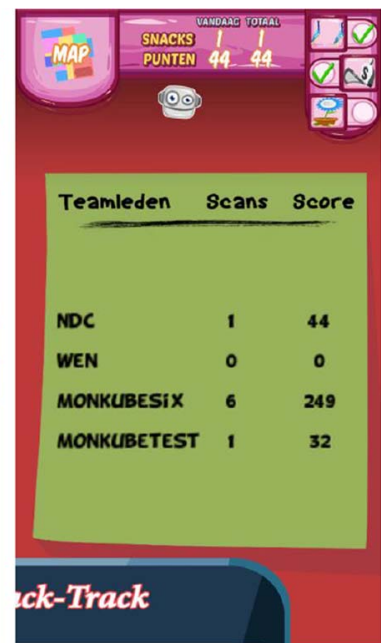

Credit system

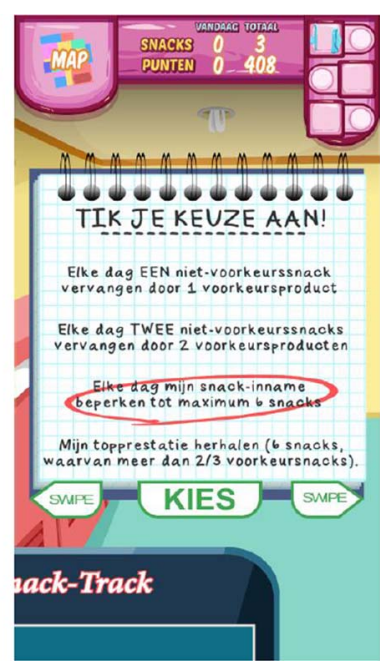

Goal-setting booklet
Fig. 3 (colour online) Screenshots of the 'Snack Track School' app's intervention components the intervention protocol ${ }^{(48)}$. Knowledge about the healthiness of snacks (proxy) was assessed by means of a scoring test. Adolescents rated the healthiness of each FFQ item (twenty-eight in total) by giving it a score ranging from 0 (very unhealthy) to 100 (very healthy). The difference between the correct score, calculated by means of the UK Ofcom NPM (rescaled to 100) ${ }^{(54)}$ (see above), and the score given by the adolescents was computed for each FFQ item. The absolute mean difference was then computed for all FFQ items; the smaller this absolute mean difference, the better their knowledge about the healthiness of snacks.

\section{Other measurements}

Adolescents' sex and age (in years) were assessed with one-item questions at baseline. The education type of the adolescents was obtained from the schools.

Height and weight were measured at baseline and postintervention by two trained research assistants using a standardized procedure ${ }^{(61)}$. Age- and sex-specific BMI $Z$-scores were calculated using Flemish 2004 growth reference data ${ }^{(62)}$. The International Obesity Task Force cut-off points were used to separate overweight and non-overweight individuals ${ }^{(63)}$.

SR was measured with the BAS Drive subscale of the Dutch version of the Carver and White BAS (behavioural activation system) scales for children ${ }^{(64)}$. In the present sample, the Cronbach's $\alpha$ for BAS Drive at baseline was $0 \cdot 80$. Scores of BAS Drive items were added and presented as a score ranging from 4 to 16 .

A more detailed explanation on how height, weight and SR were measured can be found elsewhere ${ }^{(47)}$.

In addition, snack availability at home; peer and parental influence; dietary restraint; pubertal status; total energy intake; meal patterns; duration and frequency of game play; general game preferences, engagement, motivations, addiction and preferences for structural game characteristics; and smartphone and tablet use were assessed $^{(47)}$. However, these variables were not considered in the present study.

\section{Process evaluation}

Following previous process evaluations of mHealth interventions in adolescents and young adults, the process evaluation focused on reach and dose received (exposure and satisfaction) ${ }^{(65-67)}$. According to Saunders et al., 'reach' refers to degree to which the intended priority audience participates in the intervention; 'exposure' refers to the extent to which the participants use the intervention; and 'satisfaction' refers to the satisfaction of the participants with the programme ${ }^{(45,47)}$. Within the current intervention, reach was evaluated as the number of adolescents who downloaded the app and exposure by the frequency of use of the app. Every time the adolescents used the app this was logged and stored in a log database, together with all actions they performed within that login session such as entering a snack consumption 
(time, type and points) or opening their locker (process evaluation log data). Adolescents' satisfaction with the app was measured after the intervention using the core module of the game experience questionnaire ${ }^{(68)}$, which measures seven dimensions of gamers' experience (competence; sensory and imaginative immersion; flow; annoyance; challenge; negative affect; positive affect). Mean scores were computed for each of the dimensions.

\section{Statistical analyses}

Data were analysed using the statistical software package Stata version $13 \mathrm{SE}$.

We compared sample characteristics between intervention and control group at baseline, using $\chi^{2}$ tests and $t$ statistics (adjusted for clustering using Stata's 'svy' command). In addition, we assessed if participant characteristics were associated with study attrition, also applying $\chi^{2}$ tests and $t$ statistics (adjusted for clustering).

We evaluated reach by reporting the number of adolescents who downloaded the app. Exposure or frequency of use was assessed by counting the number of days that adolescents logged into the app and ranged from 1 to 28 . Multiple logins per day were recoded to 1 for that day. The number of participants who logged into the app each day (1 to 28) of the intervention was then computed and reported. In addition, adolescents were divided in three groups according to their exposure to the app. These three 'app use' categories were created based on the continuous frequency of use, resulting in three equal app use categories (tertiles): $1=$ app non-users (logged in $\leq 0 \mathrm{~d}$ ), $2=$ low app users (logged in $<4 \mathrm{~d}$ ) and $3=$ high app users (logged in $>4 \mathrm{~d}$ ). Baseline characteristics of these non-users, high and low app users were compared using $F$ tests and $\chi^{2}$ tests adjusted for clustering (using Stata's 'svy' command). We also compared post-intervention app satisfaction ratings (competence, immersion, flow, annoyance, challenge, positive and negative affect) for the high and low app users by means of $t$ statistics (adjusted for clustering).

We assessed the intervention effect on the healthy snack ratio using multilevel linear regression modelling with three levels to account for the clustered design of the study (adolescents within classes and schools). Because of the non-random allocation of the intervention to schools we analysed the intervention effect by difference-in-difference (DID) analysis, in which the average difference in the intervention group is compared with the average difference in the control group to determine the intervention effect ${ }^{(69)}$. We conducted our analyses on the full analysis set, but also assessed impacts by exposure level as an exploratory analysis (see further). The dependent variables were the difference between post-intervention (T1) and baseline (T0) in healthy snack ratio, awareness, intention to eat healthy snacks, attitude regarding the taste of healthy snacks (attitude taste), attitude regarding overall health when consuming healthy snacks (attitude health), self-efficacy to eat healthy snacks, habit to eat healthy snacks and knowledge about the healthiness of snacks. Random effects in the models were school and class and fixed effects were a dichotomous variable indicating intervention $(=1)$ or control $(=0)$ and the baseline covariates age, BMI $Z$-score, sex and education type of the adolescents. The latter are known covariates in healthy eating interventions in children and adolescents. In these models the $b$ coefficient should be interpreted as the difference between the intervention and control group in mean change in the dependent variables from pre to post. To assess the effect of the adjusting, we also analysed the effect of the intervention using crude models.

Furthermore, we assessed if the intervention effect differed according to exposure level (exploratory) by means of the same approach as stated above for the general intervention effects, but with a categorical exposure variable with four groups $(0=$ control, $1=$ app non-users, $2=$ low app users, $3=$ high app users) as independent variable.

Finally, we explored the moderation of the intervention effects by SR and sex for all dependent variables using the above-described multilevel impact analysis, by adding respectively SR and the interaction terms $\mathrm{SR} \times$ intervention, sex $\times$ intervention and sex $\times$ SR $\times$ intervention to the adjusted models. In case of indications of moderation, analyses were run again for boys and girls separately.

For all multilevel regression models, continuous parameters were centred around the mean and outliers were removed if their values were larger or smaller than $3 \mathrm{SD}$ of the distribution. Unstandardized coefficients and their standard errors were displayed and associations with $P$ values $<0.05$ were considered statistically significant. All statistical tests were two-sided.

\section{Results}

\section{Participants}

Of the 1463 adolescents selected to participate, 681 ( $46.5 \%$ ) were part of the intervention group and 782 of the control group (see Fig. 1). Of these 1463 adolescents, 1212 successfully completed the baseline survey, with respectively 522 adolescents $(76.7 \%)$ in the intervention group and $690(88.2 \%)$ in the control group. An overview of the non-participating adolescents can be found in Fig. 1, the CONSORT flowchart.

The post-survey was completed by 416 and 572 adolescents in the intervention and control group, respectively. From baseline ( $n$ 1212) to post intervention ( $n$ 988), 106 adolescents in the intervention group and 118 in the control group dropped out (see Fig. 1). The adolescents who dropped out were significantly older $(t=3 \cdot 37$, $P<0.05)$, had a lower score for attitude regarding overall health when eating healthy snacks $(t=-3.69, P<0.05)$ and a lower knowledge about the healthiness of snacks $(t=3 \cdot 35, P<0 \cdot 05)$. No significant differences between the adolescents who dropped out and those who did not were 
found for sex, education, SR, BMI $Z$-score, healthy snack ratio, awareness, intention to eat healthy, attitude regarding the taste of healthy snacks, self-efficacy to eat healthy and habit to eat healthy snacks.

Of the 1463 adolescents, 988 completed both the baseline and post-survey and a participation rate of $67.5 \%$ was thus obtained to evaluate the intervention impact. No schools (clusters) were lost in the intervention or control group. The mean age of the 988 adolescents considered for analysis was 14.9 (sD 0.70) years, the mean BMI $Z$-score was 0.11 (sD 0.99), 59.4\% were boys, $31 \cdot 8 \%$ followed general education, $48.6 \%$ technical education and $18.4 \%$ vocational education. Table 2 shows the mean healthy snack ratio and other characteristics at baseline of the sample ( $n$ 988). No statistically significant differences were observed between the intervention and control groups at baseline. However, we note that the healthy snack ratio was $\sim 8.5 \%$ higher in the control group compared with the intervention group.

\section{Process evaluation}

\section{Reach}

In the intervention group, 268 adolescents (64.4\%) downloaded the app or borrowed a smartphone with the app already installed on it; 148 adolescents were absent at the day of installation, did not want to participate any more or could not download the app on their smartphone. These latter adolescents also did not want to borrow a smartphone with the app already installed on it.

\section{Exposure to the intervention}

Of the 268 adolescents who downloaded the app or borrowed a smartphone with the app already installed on it, 266 (99.2\%) logged in at least once in week 1, 152 (56.7\%) in week 2 , eighty-nine $(33.2 \%)$ in week 3 and fifty-five
(20.5\%) in week 4 . The percentage of adolescents who logged in at each day of the intervention decreased gradually from day 1 until day 28 (Fig. 4). Small increases around days 8, 10, 15 and 22 coincided with the days of the researchers' weekly visits.

The mean exposure to the intervention, measured in the number of days that the adolescents logged into the app, was 4.78 (SD 6.21) d for the full intervention group ( $n$ 416). When we excluded the adolescents who did not use the app ( $n$ 148), the mean exposure was $7 \cdot 41$ (sD 6.35) $\mathrm{d}$.

Non-users, low and high app users differed at baseline in age, BMI $Z$-score, SR, percentages following general, technical or vocational education, healthy snack ratio and self-efficacy to eat healthy (see Table 3 ). The high app users were the oldest with a mean age 15.03 (SD 0.04) years and followed more general education. Adolescents in this high app user group also had the highest healthy snack ratio and the highest score for self-efficacy to eat healthy and the lowest SR score at baseline. The low app users had the lowest BMI $Z$-score compared with the non-users and high users. No significant differences between non-users, low and high app users could be observed for percentage boys, awareness, intention to eat healthy, attitude regarding the taste of healthy snacks, attitude regarding overall health when eating healthy snacks, habit to eat healthy and knowledge about the healthiness of snacks.

\section{Satisfaction}

Both the high and low app users provided low rates for flow due to the app, the competence to use the app, the sensory and imaginative immersion into the app, the positive affect due to the app, the annoyance with the app and the challenge experienced (mean score $\leq 1$ 'slightly'). Both user groups did experience moderate negative affect due to the app ( 1 'slightly' < mean score $\leq 2$ 'moderately'). The high app users significantly rated the flow due to the

Table 2 Characteristics of the sample of 14-16-year-old adolescents ( $n$ 988) from six secondary schools (three intervention schools, three control schools) in two (matched) cities, 'Snack Track School' app intervention, Flanders, Belgium, January-April 2016

\begin{tabular}{|c|c|c|c|c|}
\hline & \multicolumn{2}{|c|}{$\begin{array}{l}\text { Control group } \\
\quad(n 572)\end{array}$} & \multicolumn{2}{|c|}{$\begin{array}{l}\text { Intervention group } \\
\qquad(n 416)\end{array}$} \\
\hline & Mean† & SD & Meant & SD \\
\hline Age (years) & 14.91 & 0.08 & 14.96 & $0 \cdot 10$ \\
\hline BMI Z-score & 0.13 & 0.04 & 0.08 & 0.06 \\
\hline Sensitivity to reward (range 4-16) & 8.65 & 0.26 & $9 \cdot 28$ & 0.11 \\
\hline Boys (\%) & \multicolumn{2}{|c|}{57.87} & \multicolumn{2}{|c|}{61.52} \\
\hline General education (\%) & \multicolumn{2}{|c|}{34.62} & \multicolumn{2}{|c|}{30.77} \\
\hline Technical education (\%) & \multicolumn{2}{|c|}{51.92} & \multicolumn{2}{|c|}{43.99} \\
\hline Vocational education (\%) & \multicolumn{2}{|c|}{13.46} & \multicolumn{2}{|c|}{$25 \cdot 24$} \\
\hline Healthy snack ratio & 43.29 & $2 \cdot 78$ & 39.88 & $5 \cdot 13$ \\
\hline Awareness (range 0-4) & $2 \cdot 10$ & 0.03 & 2.02 & 0.06 \\
\hline Intention (range 1-5) & 3.43 & 0.09 & 3.25 & 0.20 \\
\hline Attitude taste (range $1-5$ ) & 3.17 & 0.02 & 2.99 & 0.09 \\
\hline Attitude health (range 1-5) & 3.70 & 0.08 & 3.64 & 0.14 \\
\hline Self-efficacy (range 1-5) & 3.56 & 0.07 & 3.42 & $0 \cdot 10$ \\
\hline Habit (range $1-5$ ) & $2 \cdot 89$ & 0.09 & $2 \cdot 82$ & 0.04 \\
\hline Knowledge about the healthiness of snacks (range $0-100$ ) & $25 \cdot 26$ & 0.66 & 25.03 & 0.30 \\
\hline
\end{tabular}

†Adjusted for clustering (three clusters per group). 


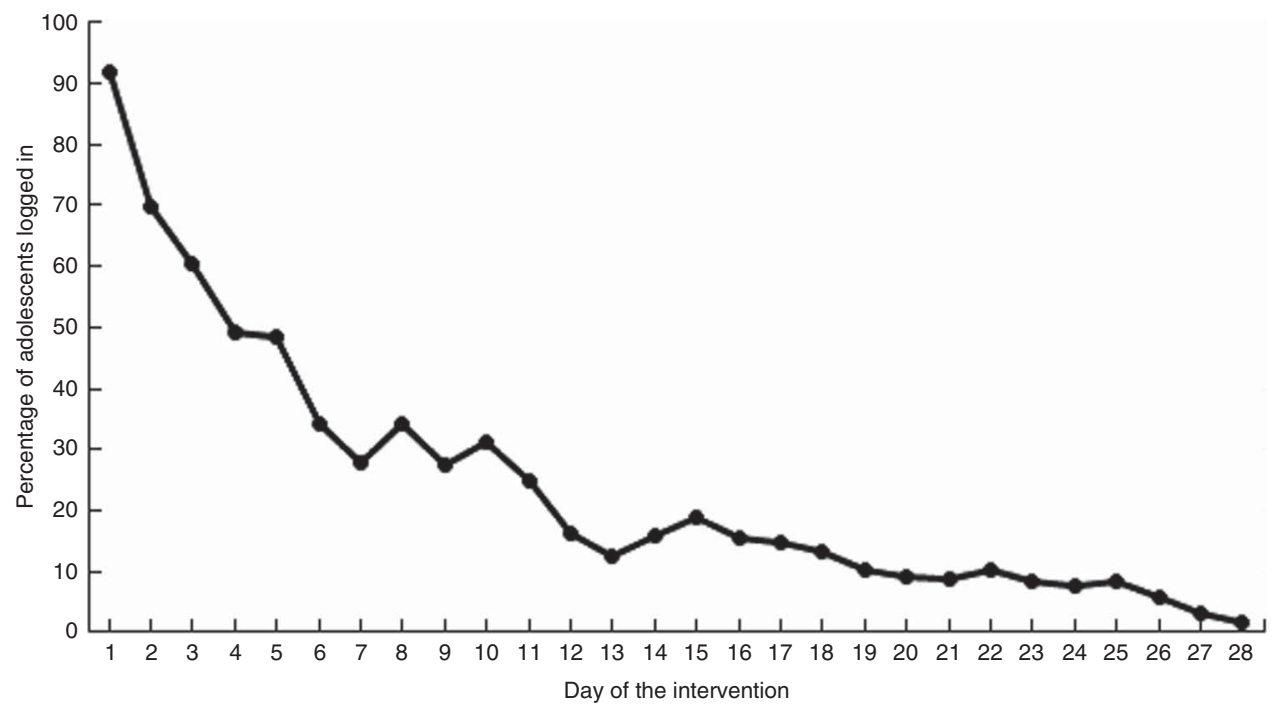

Fig. 4 Percentage of adolescents (intervention group only, $n$ 268) who logged into the app on each day of the intervention, 'Snack Track School' app intervention, Flanders, Belgium, January-April 2016

Table 3 Baseline characteristics according to app user group (intervention group only, $n$ 416) among 14-16-year-old adolescents, 'Snack Track School' app intervention, Flanders, Belgium, January-April 2016

\begin{tabular}{|c|c|c|c|c|c|c|}
\hline & \multicolumn{2}{|c|}{$\begin{array}{l}\text { App non-users } \\
(n 148)\end{array}$} & \multicolumn{2}{|c|}{$\begin{array}{l}\text { Low app users } \\
\quad(n 123)\end{array}$} & \multicolumn{2}{|c|}{$\begin{array}{l}\text { High app users } \\
\quad(n 145)\end{array}$} \\
\hline & Mean† & SD & Mean† & SD & Mean & sD $†$ \\
\hline Number of days logged in (range $0-28$ ) & 0 & 0 & $2 \cdot 38$ & 0.05 & $11.68^{\star \star \star}$, & 0.32 \\
\hline Age (years) & 14.99 & 0.20 & 14.85 & 0.09 & $15 \cdot 03^{\star \star \star}$ & 0.04 \\
\hline BMI Z-score & 0.14 & 0.40 & -0.02 & 0.08 & $0.09^{\star \star}$ & 0.08 \\
\hline Sensitivity to reward (range $4-16$ ) & 9.59 & 0.23 & 9.42 & 0.28 & $8 \cdot 84^{*}$ & 0.05 \\
\hline Boys (\%) & \multicolumn{2}{|c|}{$66 \cdot 2$} & \multicolumn{2}{|c|}{65.9} & \multicolumn{2}{|c|}{62.6} \\
\hline General education (\%) & \multicolumn{2}{|c|}{11.5} & \multicolumn{2}{|c|}{28.5} & \multicolumn{2}{|c|}{$52 \cdot 4^{\star \star}, \S$} \\
\hline Technical education (\%) & \multirow{2}{*}{\multicolumn{2}{|c|}{$\begin{array}{l}50 \cdot 7 \\
37.8\end{array}$}} & \multirow{2}{*}{\multicolumn{2}{|c|}{$\begin{array}{l}44.7 \\
26.8\end{array}$}} & \multicolumn{2}{|c|}{$36 \cdot 6^{\star *}, \S$} \\
\hline Vocational education (\%) & & & & $26 \cdot 8$ & \multicolumn{2}{|c|}{$11 \cdot 0^{\star *}, \S$} \\
\hline Healthy snack ratio & $35 \cdot 70$ & 3.65 & 38.85 & 6.54 & $45 \cdot 02^{\star \star \star}$ & $3 \cdot 12$ \\
\hline Awareness (range 0-4) & 1.98 & 0.08 & 2.02 & 0.03 & 2.05 & 0.08 \\
\hline Intention (range $1-5$ ) & $3 \cdot 19$ & 0.20 & $3 \cdot 13$ & 0.27 & 3.40 & 0.09 \\
\hline Attitude taste (range $1-5$ ) & 3.01 & 0.14 & $2 \cdot 96$ & 0.12 & 3.00 & 0.01 \\
\hline Attitude health (range $1-5$ ) & 3.50 & 0.24 & 3.69 & 0.06 & 3.73 & 0.06 \\
\hline Self-efficacy (range 1-5) & 3.31 & 0.09 & 3.45 & 0.09 & $3 \cdot 50^{\star \star}$ & $0 \cdot 10$ \\
\hline Habit (range 1-5) & $2 \cdot 82$ & 0.13 & $2 \cdot 84$ & 0.10 & 2.79 & 0.05 \\
\hline Knowledge about the healthiness of snacks (range $0-100$ ) & 26.09 & 0.92 & $25 \cdot 41$ & 0.38 & 23.68 & 0.88 \\
\hline
\end{tabular}

${ }^{\star} P<0.05,{ }^{* \star} P<0.01,{ }^{* \star *} P<0.00$.

†Adjusted for clustering.

$\ddagger t$ Test for low and high app users.

$\S$ Same $x^{2}$ test for the variable education type.

app lower, felt more competent to use the app and experienced more positive affect due to the app than the low app users (see Table 4). No significant differences between high and low app users were observed for immersion, annoyance, challenge and negative affect.

\section{Effect evaluation}

Overall effects on the primary and secondary outcomes We did not find statistically significant differences between the intervention and control groups for the healthy snack ratio, awareness, intention to eat healthy, attitude regarding the taste of healthy snacks, self-efficacy to eat healthy and habit to eat healthy snacks (see Table 5). A significant difference between intervention and control groups was observed for attitude regarding overall health when eating healthy snacks and knowledge about the healthiness of snacks. The score for attitude regarding overall health when eating healthy snacks decreased from baseline (T0) to post-intervention (T1) with 0.13 (SE 0.05) points more ( $P=0 \cdot 0$, Cohen's $d=0 \cdot 16$ ) in the intervention group than in the control group. The knowledge about the healthiness of snacks decreased from T0 to T1 in the intervention group with 1.37 (SE 0.25 ) points $(P=0.04$, Cohen's $d=0.20)$ compared with the control group, where the knowledge increased. 
Intervention effects according to exposure groups

A difference between the control group and the low app user group was observed for attitude regarding overall health when eating healthy snacks (Table 6). The low app users had a significantly higher decrease in attitude compared with the control group $(b=-0.24$ (sE 0.08), $P<0 \cdot 01)$. A difference between the control group and the non-users and low app users was also observed for the knowledge about the healthiness of snacks (Table 6). The non-users and low app users had a higher decrease in knowledge about the healthiness of snacks compared with the control group $(b=1.66$ (sE 0.71$), P<0.05$ for nonusers; $b=1.55$ (se 0.72 ), $P<0.05$ for the low app users). No other significant differences were observed between the control group and the high app users.

\section{Moderation analysis}

A significant three-way interaction effect (intervention $\times$ SR $\times$ sex) was found for difference in healthy snack ratio $(b=-3.92$ (sE 1.33), $P<0.01)$. When analyses were

Table 4 App satisfaction ratings for high and low app users (intervention group only, $n$ 416) among 14-16-year-old adolescents, 'Snack Track School' app intervention, Flanders, Belgium, January-April 2016

\begin{tabular}{lccllll}
\hline & \multicolumn{2}{c}{$\begin{array}{c}\text { Low app users } \\
(n \text { 123) }\end{array}$} & & \multicolumn{2}{c}{$\begin{array}{c}\text { High app users } \\
(n \text { 145) }\end{array}$} \\
\cline { 2 - 3 } & Mean† & SD & & & Mean† & SD \\
\hline Competence (range 0-4) & 0.72 & 0.07 & & $1.04^{* *}$ & 0.08 \\
Immersion (range 0-4) & 0.46 & 0.06 & & 0.48 & 0.06 \\
Flow (range 0-4) & 0.36 & 0.11 & & $0.20^{\star}$ & 0.08 \\
Annoyance (range 0-4) & 0.96 & 0.02 & & 0.86 & 0.04 \\
Challenge (range 0-4) & 0.63 & 0.06 & & 0.51 & 0.01 \\
Negative affect (range 0-4) & 2.01 & 0.05 & & 1.99 & 0.06 \\
Positive affect range (0-4) & 0.62 & 0.10 & & $0.76^{*}$ & 0.10
\end{tabular}

${ }^{*} P<0.05,{ }^{* *} P<0.01$.

†Adjusted for clustering. conducted separately for boys and girls, a significant and contrasting intervention $\times$ SR interaction was found for both (boys: $b=1.92$ (se 0.81), $P<0.05$; girls: $b=-2.28$ (SE 1.02), $P<0.05$ ). Margin plots are shown in Fig. 5. In boys of the intervention group, the intervention increased the healthy snack ratio with higher SR ( $b=1.38$ (SE 0.59), $P<0.05)$, whereas in girls the opposite was observed $(b=-1.90$ (sE 0.94), $P<0.05)$. In the control group the healthy snack ratio did not increase or decrease significantly from T0 to T1 with higher SR in boys or girls.

\section{Discussion}

The present study evaluated the feasibility and impact of a newly developed smartphone app 'Snack Track School' on the healthy snack ratio and the targeted determinants of Flemish adolescents aged 14-16 years old. The intervention incorporated rewarding strategies together with reflective strategies delivered through a gamified application. We were unable to demonstrate a significant positive impact of the intervention on the healthy snack ratio and targeted determinants compared with the control group. The process evaluation results, however, allow us to better understand these findings.

The reach of and exposure to the intervention was low. As for reach, only $64.4 \%$ of the adolescents in the intervention group downloaded the app. This could be explained by the difficult installation process of the app. The installation of the app was time-consuming and required considerable smartphone memory. The percentage of adolescents who used the app (exposure) also decreased gradually over the intervention period. Of the 268 adolescents who actually used the app, only $20.5 \%$ were still logging into it in the fourth week of the intervention. This low engagement could possibly be

Table 5 Effect of the intervention on the difference in outcomes between T0 (baseline) and T1 (post-intervention) among 14-16-year-old adolescents, 'Snack Track School' app intervention, Flanders, Belgium, January-April 2016

\begin{tabular}{|c|c|c|c|c|c|c|c|c|c|c|}
\hline \multirow[b]{2}{*}{ Outcome } & \multicolumn{4}{|c|}{ Difference } & \multicolumn{2}{|c|}{ Unadjusted effects $†$} & \multicolumn{2}{|c|}{ Adjusted effects $\ddagger$} & \multicolumn{2}{|c|}{ Effect size } \\
\hline & $\Delta \mathrm{C} \S$ & SD & $\Delta \mathrm{I} \S$ & SD & DID & SE & DID & SE & Cohen's $d \|$ & Cohen's $f^{2} \boldsymbol{q}$ \\
\hline Healthy snack ratio & 3.38 & 0.23 & 1.28 & 1.31 & $-2 \cdot 27$ & 1.80 & -3.52 & 1.82 & -0.139 & 0.000 \\
\hline Awareness & 0.02 & 0.01 & 0.04 & 0.00 & 0.04 & 0.06 & 0.04 & 0.06 & 0.046 & 0.001 \\
\hline Intention & -0.08 & 0.06 & -0.23 & 0.02 & -0.14 & 0.08 & -0.12 & 0.07 & -0.114 & 0.000 \\
\hline Attitude taste & -0.19 & 0.05 & -0.16 & 0.05 & 0.07 & 0.07 & 0.10 & 0.08 & 0.089 & 0.002 \\
\hline Attitude health & -0.17 & 0.03 & -0.32 & 0.02 & -0.14 & $0.05^{\star}$ & $-0.13^{\star}$ & 0.05 & $-0 \cdot 160$ & 0.004 \\
\hline Self-efficacy & -0.00 & 0.04 & -0.07 & 0.05 & -0.05 & 0.08 & -0.05 & 0.06 & -0.427 & 0.000 \\
\hline Habit & 0.04 & 0.05 & -0.00 & 0.02 & -0.03 & 0.06 & 0.00 & 0.06 & 0.001 & 0.000 \\
\hline Knowledge about the healthiness of snacks & -0.12 & 0.23 & 1.16 & 0.26 & $1.35^{\star \star}$ & 0.47 & $1.37^{\star \star}$ & 0.25 & 0.200 & 0.003 \\
\hline
\end{tabular}

$\Delta \mathrm{C}$, mean difference of the outcomes measured before and after the intervention in the control group; $\Delta \mathrm{l}$, mean difference of the outcomes measured before and after the intervention in the intervention group; DID, difference-in-difference.

${ }^{\star} P<0.05,{ }^{\star *} P<0.01$.

†Crude multilevel models without covariates.

$\ddagger$ Multilevel models adjusted for age, BMl Z-score, sex and education type.

$\S$ Adjusted for clustering.

|Cohen's $d$ was calculated by dividing the adjusted DID coefficient by the total residual variance ${ }^{(86,87)}$.

ๆCohen's $f^{2}$ was calculated as follows ${ }^{(88)}$ : $\left(R^{2}\right.$ full model $-R^{2}$ reduced model $) /\left(1-R^{2}\right.$ reduced model). 
Table 6 Effect of the exposure on the difference in healthy snack ratio and the targeted determinants between T0 (baseline) and T1 (post-intervention), as compared with the control group, among 14-16-year-old adolescents, 'Snack Track School' app intervention, Flanders, Belgium, January-April 2016

\begin{tabular}{|c|c|c|c|c|}
\hline & \multicolumn{2}{|c|}{ Unadjusted effects $†$} & \multicolumn{2}{|c|}{ Adjusted effects $¥$} \\
\hline & DID & SE & DID & SE \\
\hline \multicolumn{5}{|l|}{ Healthy snack ratio } \\
\hline \multicolumn{5}{|l|}{ Exposure§ } \\
\hline Did not use the app & -0.28 & 2.48 & $-3 \cdot 33$ & $2 \cdot 66$ \\
\hline Low users & $-3 \cdot 21$ & $2 \cdot 64$ & -3.35 & $2 \cdot 74$ \\
\hline High users & -3.42 & 2.50 & -3.80 & 2.54 \\
\hline \multicolumn{5}{|l|}{ Awareness } \\
\hline \multicolumn{5}{|l|}{ Exposure§ } \\
\hline Did not use the app & -0.01 & 0.08 & -0.03 & 0.09 \\
\hline Low users & 0.10 & 0.08 & 0.15 & 0.09 \\
\hline High users & -0.01 & 0.04 & 0.01 & 0.08 \\
\hline \multicolumn{5}{|l|}{ Intention } \\
\hline \multicolumn{5}{|l|}{ Exposure§ } \\
\hline Did not use the app & $-0.21^{*}$ & $0 \cdot 10$ & $-0 \cdot 16$ & $0 \cdot 11$ \\
\hline Low users & $-0 \cdot 16$ & 0.10 & -0.08 & 0.11 \\
\hline High users & $-0 \cdot 10$ & 0.10 & -0.11 & $0 \cdot 10$ \\
\hline \multicolumn{5}{|l|}{ Attitude taste } \\
\hline \multicolumn{5}{|l|}{ Exposure§ } \\
\hline Did not use the app & -0.06 & $0 \cdot 10$ & 0.08 & 0.11 \\
\hline Low users & 0.01 & 0.11 & $0 \cdot 10$ & 0.12 \\
\hline High users & $0 \cdot 16$ & $0 \cdot 10$ & $0 \cdot 12$ & 0.11 \\
\hline \multicolumn{5}{|l|}{ Attitude health } \\
\hline \multicolumn{5}{|l|}{ Exposure§ } \\
\hline Did not use the app & $-0 \cdot 16^{\star}$ & 0.07 & $-0 \cdot 10$ & 0.08 \\
\hline Low users & $-0.26^{\star \star}$ & 0.08 & $-0 \cdot 24^{\star \star}$ & 0.08 \\
\hline High users & -0.05 & 0.07 & -0.07 & 0.07 \\
\hline \multicolumn{5}{|l|}{ Self-efficacy } \\
\hline \multicolumn{5}{|l|}{ Exposure§ } \\
\hline Did not use the app & $-0 \cdot 10$ & 0.08 & -0.09 & 0.09 \\
\hline Low users & -0.12 & 0.09 & -0.10 & 0.09 \\
\hline High users & 0.04 & 0.08 & 0.03 & 0.08 \\
\hline \multicolumn{5}{|l|}{ Habit } \\
\hline \multicolumn{5}{|l|}{ Exposure§ } \\
\hline Did not use the app & -0.02 & 0.08 & 0.05 & 0.08 \\
\hline Low users & -0.13 & 0.08 & -0.08 & 0.08 \\
\hline High users & 0.02 & 0.08 & 0.02 & 0.08 \\
\hline \multicolumn{5}{|c|}{ Knowledge about the healthiness of snacks } \\
\hline \multicolumn{5}{|c|}{ Exposure§ } \\
\hline Did not use the app & 1.44 & $0.67^{*}$ & $1.66^{*}$ & 0.71 \\
\hline Low users & 1.46 & $0.71^{*}$ & $1.55^{\star}$ & 0.72 \\
\hline High users & 1.02 & 0.67 & 1.01 & 0.67 \\
\hline
\end{tabular}

DID, difference-in-difference.

${ }^{*} P<0.05,{ }^{* *} P<0.01$.

†Crude multilevel models without covariates.

$\ddagger$ Multilevel models adjusted for age, BMI Z-score, sex and education type.

$\S$ Reference group $=$ control group.

explained by the low app satisfaction. Mean ratings of app satisfaction were low in both the low and the high app user groups. The adolescents reported to experience little flow, a mental state characterized by focused attention and enjoyment $^{(70)}$, challenge and positive feelings when playing the app. Despite our efforts to develop attractive game components in participation with the target population (see above), the app was insufficiently engaging for the adolescents. Efforts will thus be needed to increase the feeling of flow and the experienced challenge with the 'Snack Track School' app to improve the engagement. Better understanding and improvement of factors that determine participant engagement and retention is crucial

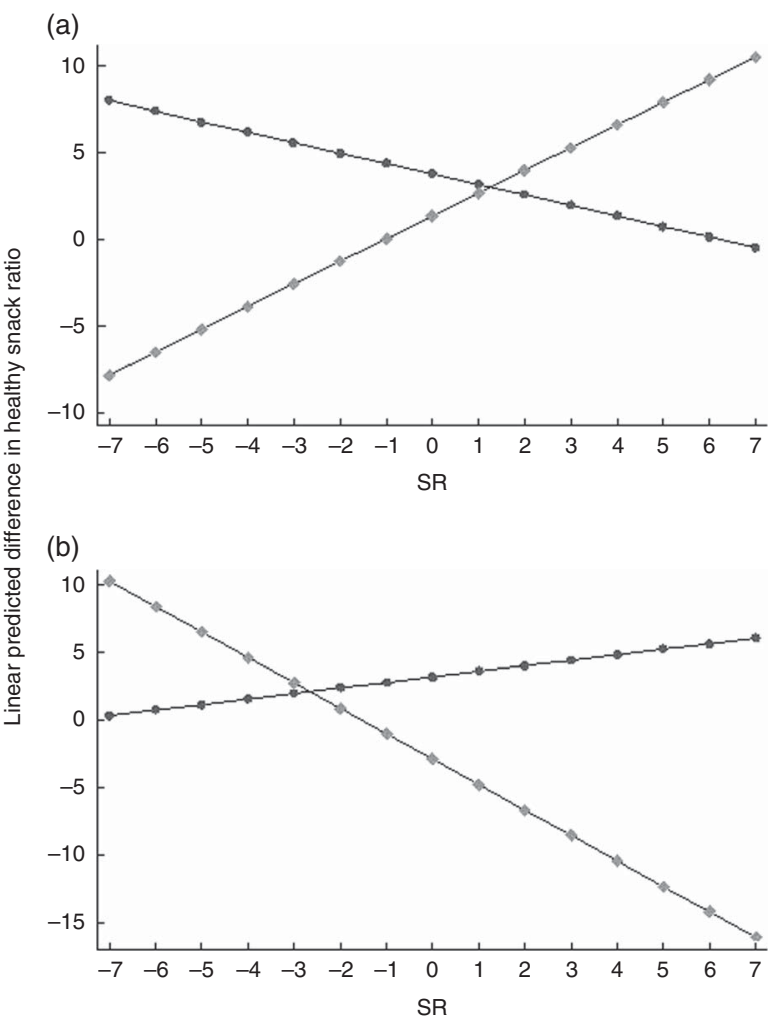

Fig. 5 Margin plots of sensitivity to reward $(\mathrm{SR}) \times$ condition (-- control group, $n 572$; - - , intervention group, $n 416$ ) on healthy snack ratio for adolescent boys (a) and girls (b), 'Snack Track School' app intervention, Flanders, Belgium, JanuaryApril 2016. Analyses controlled for age, BMI Z-score and education type

to improve intervention impact ${ }^{(42,70,71)}$. Engagement with digital behaviour change interventions is influenced by the used features ${ }^{(70)}$; given that the current app intervention was a combination of rewarding strategies, reflective strategies and game mechanisms, further exploration of the log data together with the collected qualitative data will be needed to determine which app features and/or behaviour change techniques mainly need to be altered in order to increase engagement.

A higher use of the app was also not related to positive intervention effects. It might be that the use of the app even within the highest app user group was inadequate to achieve the desired effects. The mean number of days that these high app users logged into the app was still only $12 \mathrm{~d}$, which is less than half of the intervention period. However, self-selection might also play a role here: the high app user group already had the highest healthy snack ratio, self-efficacy to eat healthy and the lowest SR at baseline.

Only a few other studies also developed an app- or web-based game to improve adolescents' health ${ }^{(66,72,73)}$. 'Diabetic Mario', a mobile game to improve diabetes management based on informal learning principles, showed positive effects on diabetes management ${ }^{(73)}$. The adolescents also enjoyed playing the game and gave 
positive satisfaction ratings ${ }^{(73)}$. However, the game was only pilot-tested in a sample of twelve adolescents, and a larger efficacy trial is yet to come. 'Balance It', an appbased intervention to promote healthy eating and higher physical activity in adolescents based on self-regulation techniques, showed positive effects only in a subgroup of high users ${ }^{(66)}$. Only $27 \cdot 6 \%$ of the adolescents actually used the app as intended and neutral to positive app satisfaction ratings were given ${ }^{(66)}$, which is comparable to the retention and satisfaction achieved in the present study. 'Creature 101', a web-based game which aimed to change energy balance-related behaviour in adolescents based on social cognitive and self-determination theory, reported an intervention retention rate of $64 \%$ and was able to significantly reduce intake of sweetened beverages and processed snacks ${ }^{(72)}$. 'Creature 101' was implemented within the school curriculum, while our 'Snack Track School' was a stand-alone intervention in which adolescents used the app with minimal external assistance or instructions during school breaks or at home. As argued earlier ${ }^{(74)}$, intervention retention and effects could possibly also be improved by embedding our app within the existing school structure. Also, teachers were currently not engaged in the intervention implementation, as school directors preferred that the teachers were not to be burdened even more. Stok et al. also mention that adolescents prefer intervention strategies to be delivered by teachers than by policy makers ${ }^{(75)}$. Also, as small increases in the percentage of adolescents logged in were observed after the visits of the researchers, giving teachers a more active role to remind or encourage the adolescents could greatly improve retention. In addition, reviews by DeSmet et $a l .{ }^{(76)}$ and Schoeppe et al. ${ }^{(42)}$ reported that intervention effects were higher for respectively serious games or apps incorporated within a multicomponent intervention. Incorporating the app in a multicomponent intervention embedded in existing school structures, such as classes, and involving the school teachers more in the intervention implementation, could thus help to increase intervention retention and impact. It could also help to decrease the initial large dropout, as the unbalanced dropout was most likely to be related to the intervention. Several parents did not want their child to enrol in a four-week mHealth intervention programme, because this would distract them too much from their schoolwork.

Evidence of moderation of the intervention effects by sex and reward sensitivity was found for the healthy snack ratio. The intervention slightly increased the health snack ratio in boys with higher SR, while in girls the opposite was observed. The latter could be interpreted by the fact that girls already ate healthier at the start of the intervention (girls had a significantly higher healthy snack ratio at baseline than boys, $t=-8.12$ and $P<0.001)$ and rewarding strategies may have had a counterproductive effect. Previous studies found that rewards can have a counterproductive effect when the food is already liked ${ }^{(26,77)}$.
However, the intervention was a combination of game features and reflective and rewarding behaviour change strategies. Also, high app users were more often female and following general education, felt more competent to use the app, had a higher attitude regarding overall health when eating healthy and had a higher healthy snack ratio at baseline. This confirms previous studies that reported a more intense use of health-related apps to be associated with being female and being higher educated ${ }^{(78,79)}$. Possibly girls used more the app's reflective methods such as the goal-setting booklet or the report card. Previous research also indicated that female children, adolescents and young adults have healthier food preferences, stronger beliefs in healthy eating and show more weight control involvement than male subjects ${ }^{(80,81)}$. The game setting and features might also have appealed more to girls than to boys. Girls tend to prefer simpler explorative games, while boys prefer competitive challenging games ${ }^{(82,83)}$. However, girls did not have higher app satisfaction ratings than boys (results not shown) in the intervention group despite the higher use. Exploration of the log data together with the collected qualitative data (see above) could also shed light on the different game features and behaviour change strategies used/preferred by girls and boys and high SR girls, high SR boys, low SR girls and low SR boys. However, the current data already indicate that different strategies and/or app features might be needed to achieve healthier snacking habits depending on sex and SR. The reviews by DeSmet et al. ${ }^{(76)}$ and Schoeppe et al. ${ }^{(42)}$ also concluded that tailoring smartphone apps to specific populations or user characteristics might enhance intervention impact.

To date, only a few other studies have assessed the effectiveness of smartphone apps to change adolescents' or children's eating or physical activity behaviors $^{(42,66,71-73)}$. To our knowledge, the present study is the only one that considered both rewarding (targeting the implicit habits driven by the difference in reinforcing value between healthy and unhealthy snacks) and reflective strategies (targeting the explicit pathways) to improve adolescents' choices of healthy snacks. In addition, only a few other studies reported to $\log$ all actions of their intervention users ${ }^{(66,73)}$. Schoeppe et al. stressed that more of such objective app usage statistics should be collected to better understand levels of engagement and reasons for participant (dis)engagement and intervention exposure $^{(42,43)}$. Another strength of the present study was the elaborate intervention development process (based on the principles of intervention mapping) that included a strong theoretical base, several preliminary studies and a participatory approach. Our study also had limitations. First, the intervention was not randomized, so selection bias could have occurred. However, we used a mixed DID model and also adjusted the analyses for baseline values of age, BMI $Z$-score, sex and education type ${ }^{(69)}$. Second, we were unable to assess if borrowing a smartphone led to 
different intervention effects. Due to practical difficulties, we were unable to keep track which adolescents completed the intervention on a borrowed smartphone. Borrowing a smartphone might have increased the app use and/or satisfaction in those not having an own smartphone, while having to carry two smartphones in those having an own smartphone might have decreased use and/or satisfaction. Given that app use itself was not associated to differences in impact, we are however fairly confident that borrowing a smartphone will not have influenced the intervention impact. Third, the possibility that participants lied about their snack intake to get more points was a limitation. This was countered, however, by the built-in snack peer validation system. At random, participants were asked to take a selfie showing that the snack entered in the app was truly being consumed. These pictures then needed to be validated by their peers in the app via the validation feature of the app. Two peers were to agree that the snack entered in the app fitted the selfie. In case two different answers were given, a project researcher took the final decision to determine if the participants had cheated or not. If the participant was considered a cheater, the given points for that snack were deducted the next day and the participant needed to complete a punishment, which consisted of a small game cleaning the playground, before being able to continue using the app. If a participant cheated, this was recorded in the log data; however, full analysis of the log data was beyond the scope of the current paper. Fourth, snacks were classified as either unhealthy or healthy using the UK Ofcom NPM. This nutrient profile model was chosen over others because it provides a continuous score, awards points based on both positive and negative constituents, is an across-the-board model, is suitable for all types of food products, evaluates all food products in the same way and was externally validated ${ }^{(84,85)}$. However, this model scores items based on the nutrient composition per $100 \mathrm{~g}$, not taking portion size into account. The latter is unfortunate, as snacks are eaten in typical portion sizes such as 'one bar', 'one bag' or 'one piece' that is sometimes larger than $100 \mathrm{~g}$ like one kebab. The portion size should thus also contribute to the evaluation of a food product as a healthy or unhealthy snack choice. However, to date, no specific nutrient profile model for snacks has been developed and therefore the best suitable model was chosen. A final limitation was that the data on snack intake and the determinants were self-reported and were thus subject to social desirability bias. It was attempted to counter this bias by emphasizing anonymity of the data collection.

\section{Conclusions}

The current 'Snack Track School' app was not able to improve adolescents' snack choices or their determinants, due to the low reach, exposure and satisfaction of the involved adolescents. However, the process evaluation raised several crucial points to improve future intervention development, retention and impact in adolescents.

First, choosing an attractive intervention medium, a gamified app, is not enough to achieve a high reach and continued engagement. In the future, intervention developers should opt to incorporate apps in multicomponent interventions embedded in existing school structures and involve school teachers in the intervention implementation.

Second, extensive attention should be paid to the content (behaviour change strategies and game features) and design of the app. Content and design more appealing and engaging to the adolescents should be chosen, longer testing with and consulting of the adolescents should be considered, and the translation of behaviour change techniques to app components should be extensively studied.

Third and finally, tailoring of the app content (based on individual characteristics) to improve impact, reach, exposure and/or satisfaction should be considered.

\section{Acknowledgements}

Acknowledgements: Data collection was assisted by several students: Annelies Malengier, Liesbeth Vandendriessche, Lotte De Vos, Sander Vandamme, Elke Rammant, Floor De Groote, Lisa Schoenmaekers, Ilka Walleyn, Jana De Block, Delphine Herman and Lisa Van Wilder. Financial support: This study was supported by the Flemish Agency for Innovation \& Entrepreneurship (Belgium). The sponsors were not involved in the study design, collection, analysis or interpretation of the data. The first and corresponding author had access to all data at all times and had the final responsibility to submit the manuscript for publication. Conflict of interest: The authors declare that there were no conflicts of interest. Authorship: The authors' responsibilities were as follows: N.D.C. conducted the research, conducted the analyses and wrote the paper; W.V.L. and C.L. helped analysing the results and writing the paper; J.V. and M.N. conducted the research and helped revise the manuscript; L.H., L.G., K.B., S.E., B.D., L.M., J.V.C., C.B., E.M. and S.V. designed the research and helped revise the manuscript. All authors read and approved the final manuscript. Ethics of buman subject participation: This study conducted according to the guidelines laid down in the Declaration of Helsinki and all procedures involving human subjects were approved by the Ethics Committee of the University Hospital of Ghent University and the University of Leuven. Passive written informed consent was obtained from the parents of the participating adolescents.

\section{References}

1. Spear BA (2002) Adolescent growth and development. J Am Diet Assoc 102, 3 Suppl., S23-S29.

2. Diethelm K, Jankovic N, Moreno LA et al. (2012) Food intake of European adolescents in the light of different 
food-based dietary guidelines: results of the HELENA (Healthy Lifestyle in Europe by Nutrition in Adolescence) Study. Public Health Nutr 15, 386-398.

3. Phillips SM, Bandini LG, Naumova EN et al. (2004) Energydense snack food intake in adolescence: longitudinal relationship to weight and fatness. Obes Res 12, 461-472.

4. Lien N, Lytle LA \& Klepp KI (2001) Stability in consumption of fruit, vegetables, and sugary foods in a cohort from age 14 to age 21. Prev Med 33, 217-226.

5. Sebastian RS, Cleveland LE \& Goldman JD (2008) Effect of snacking frequency on adolescents' dietary intakes and meeting national recommendations. $J$ Adolesc Health 42, 503-511.

6. Bucher T, Collins C, Diem S et al. (2016) Adolescents' perception of the healthiness of snacks. Food Qual Prefer 50, 94-101.

7. De Vet E, Stok FM, De Wit JBF et al. (2015) The habitual nature of unhealthy snacking: how powerful are habits in adolescence? Appetite 95, 182-187.

8. Rodriguez G \& Moreno LA (2006) Is dietary intake able to explain differences in body fatness in children and adolescents? Nutr Metab Cardiovasc Dis 16, 294-301.

9. De Bourdeaudhuij I, Van Cauwenberghe E, Spittaels $\mathrm{H}$ et al. (2011) School-based interventions promoting both physical activity and healthy eating in Europe: a systematic review within the HOPE project. Obes Rev 12, 205-216.

10. Stice E, Shaw H \& Marti CN (2006) A meta-analytic review of obesity prevention programs for children and adolescents: the skinny on interventions that work. Psychol Bull 132, 667-691.

11. Kremers SP, de Bruijn GJ, Visscher TL et al. (2006) Environmental influences on energy balance-related behaviors: a dual-process view. Int J Behav Nutr Phys Act 3, 9.

12. Deutsch R \& Strack F (2006) Duality models in social psychology: from dual processes to interacting systems. Psychol Inq 17, 166-172.

13. Hagger MS (2016) Non-conscious processes and dualprocess theories in health psychology. Health Psychol Rev 10, 375-380.

14. Rothman AJ, Sheeran P \& Wood W (2009) Reflective and automatic processes in the initiation and maintenance of dietary change. Ann Behav Med 38, Suppl. 1, S4-S17.

15. van't Riet J, Sijtsema SJ, Dagevos H et al. (2011) The importance of habits in eating behaviour. An overview and recommendations for future research. Appetite 57, $585-596$.

16. Reinaerts E, de Nooijer J, Candel M et al. (2007) Explaining school children's fruit and vegetable consumption: the contributions of availability, accessibility, exposure, parental consumption and habit in addition to psychosocial factors. Appetite 48, 248-258.

17. Michie S, Abraham C, Whittington C et al. (2009) Effective techniques in healthy eating and physical activity interventions: a meta-regression. Health Psychol 28, 690-701.

18. Carver CS \& Scheier MF (1982) Control theory: a useful conceptual framework for personality-social, clinical, and health psychology. Psychol Bull 92, 111-135.

19. Appelhans BM, Woolf K, Pagoto SL et al. (2011) Inhibiting food reward: delay discounting, food reward sensitivity, and palatable food intake in overweight and obese women. Obesity (Silver Spring) 19, 2175-2182.

20. Goldfield GS, Lumb AB \& Colapinto CK (2011) Relative reinforcing value of energy-dense snack foods in overweight and obese adults. Can J Diet Pract Res 72, 170-174.

21. Goldfield GS \& Epstein LH (2002) Can fruits and vegetables and activities substitute for snack foods? Health Psychol 21, 299-303.

22. Vervoort L, Clauwaert A, Vandeweghe L et al. (2017) Factors influencing the reinforcing value of fruit and unhealthy snacks. Eur J Nutr 56, 2589-2598.
23. Hennegan JM, Loxton NJ \& Mattar A (2013) Great expectations. Eating expectancies as mediators of reinforcement sensitivity and eating. Appetite 71, 81-88.

24. Horstmann A, Dietrich A, Mathar D et al. (2015) Slave to habit? Obesity is associated with decreased behavioural sensitivity to reward devaluation. Appetite $\mathbf{8 7}$, 175-183.

25. Ames SL, Kisbu-Sakarya Y, Reynolds KD et al. (2014) Inhibitory control effects in adolescent binge eating and consumption of sugar-sweetened beverages and snacks. Appetite 81, 180-192.

26. Vandeweghe L, Verbeken S, Moens E et al. (2016) Strategies to improve the willingness to taste: the moderating role of children's reward sensitivity. Appetite 103, 344-352.

27. Wardle J, Herrera ML, Cooke L et al. (2003) Modifying children's food preferences: the effects of exposure and reward on acceptance of an unfamiliar vegetable. Eur J Clin Nutr 57, 341-348.

28. Lowe CF, Horne PJ, Hardman CA et al. (2006) A peermodeling and rewards-based intervention is effective in increasing fruit and vegetable consumption in children. Prev Med 43, 351-351.

29. Cooke LJ, Chambers LC, Anez EV et al. (2011) Eating for pleasure or profit: the effect of incentives on children's enjoyment of vegetables. Psychol Sci 22, 190-196.

30. Nederkoorn C, Braet C, Van Eijs Y et al. (2006) Why obese children cannot resist food: the role of impulsivity. Eat Behav 7, 315-322.

31. Blissett J, Bennett C, Fogel A et al. (2016) Parental modelling and prompting effects on acceptance of a novel fruit in 2-4-year-old children are dependent on children's food responsiveness. Br J Nutr 115, 554-564.

32. Davis C, Patte K, Levitan R et al. (2007) From motivation to behaviour: a model of reward sensitivity, overeating, and food preferences in the risk profile for obesity. Appetite $\mathbf{4 8}$, 12-19.

33. Dietrich A, Federbusch M, Grellmann C et al. (2014) Body weight status, eating behavior, sensitivity to reward/punishment, and gender: relationships and interdependencies. Front Psychol 5, 1073.

34. Gray JA (1994) Three fundamental emotion systems. In The Nature of Emotion: Fundamental Questions, pp. 243-247 [P Ekman and RJ Davidson, editors]. New York: Oxford University Press.

35. Verbeken S, Braet C, Lammertyn J et al. (2012) How is reward sensitivity related to bodyweight in children? Appetite 58, 478-483.

36. De Cock N, Van Lippevelde W, Vervoort L et al. (2016) Sensitivity to reward is associated with snack and sugarsweetened beverage consumption in adolescents. Eur $J$ Nutr 55, 1623-1632.

37. Apestaartjaren (2016) Onderzoeksrapport Apestaartjaren 6. https://www.apestaartjaren.be/onderzoek/apestaartjaren-6 (accessed March 2018).

38. Schoffman DE, Turner-McGrievy G, Jones SJ et al. (2013) Mobile apps for pediatric obesity prevention and treatment, healthy eating, and physical activity promotion: just fun and games? Transl Behav Med 3, 320-325.

39. Brannon EE \& Cushing CC (2015) A systematic review: is there an app for that? Translational science of pediatric behavior change for physical activity and dietary interventions. J Pediatr Psychol 40, 373-384.

40. Burrows TL, Khambalia AZ, Perry R et al. (2015) Great 'appeal' but not there yet: a review of iPhone nutrition applications relevant to child weight management. Nutr Diet 72, 363-367.

41. Dute DJ, Bemelmans WJ \& Breda J (2016) Using mobile apps to promote a healthy lifestyle among adolescents and students: a review of the theoretical basis and lessons learned. JMIR Mhealth Uhealth 4, e39. 
42. Schoeppe S, Alley S, Van Lippevelde W et al. (2016) Efficacy of interventions that use apps to improve diet, physical activity and sedentary behaviour: a systematic review. Int J Behav Nutr Phys Act 13, 127.

43. Partridge SR, Juan SJ, McGeechan K et al. (2015) Poor quality of external validity reporting limits generalizability of overweight and/or obesity lifestyle prevention interventions in young adults: a systematic review. Obes Rev 16, 13-31.

44. Moore GF, Audrey S, Barker M et al. (2015) Process evaluation of complex interventions: Medical Research Council guidance. BMJ 350, h1258.

45. Saunders RP, Evans MH \& Joshi P (2005) Developing a process-evaluation plan for assessing health promotion program implementation: a how-to guide. Health Promot Pract 6, 134-147.

46. REWARD Project (2013) REWARD study homepage. http:// www.rewardstudy.be (accessed September 2016).

47. Van Lippevelde W, Vangeel J, De Cock N et al. (2016) Using a gamified monitoring app to change adolescents' snack intake: the development of the REWARD app and evaluation design. BMC Public Health 16, 725.

48. Schulz KF, Altman DG \& Moher D (2010) CONSORT 2010 statement: updated guidelines for reporting parallel group randomised trials. BMJ 340, c332.

49. Des Jarlais DC, Lyles C, Crepaz N et al. (2004) Improving the reporting quality of nonrandomized evaluations of behavioral and public health interventions: the TREND statement. Am J Public Health 94, 361-366.

50. Konstantopoulos S (2008) The power of the test for treatment effects in three-level cluster randomized designs.J Res Educ Effect 1, 66-88.

51. De Cock N, Van Camp J, Kolsteren P et al. (2017) Development and validation of a quantitative snack and beverage FFQ for adolescents. J Hum Nutr Diet 30, 141-150.

52. Bartholomew LK, Parcel GS, Kok G et al. (2011) Planning Health Promotion Programs: An Intervention Mapping Approach, 3rd ed. San Francisco; CA: Jossey-Bass.

53. Nubel vZw (2015) Database of Tradenames. Brussels: Nubel vzw.

54. Department of Health (2011) Nutrient Profiling Technical Guidance. https://www.gov.uk/government/publications/thenutrient-profiling-model (accessed March 2018).

55. Evans EW, Jacques PF, Dallal GE et al. (2015) The role of eating frequency on total energy intake and diet quality in a low-income, racially diverse sample of schoolchildren. Public Health Nutr 18, 474-481.

56. Llaurado E, Albar SA, Giralt M et al. (2016) The effect of snacking and eating frequency on dietary quality in British adolescents. Eur J Nutr 55, 1789-1797.

57. Vlaams Institut Gezond Leven (2014) Vigez aanbevelingen voor voeding voor jongeren. https://www.gezondleven.be/ themas/voeding/aanbevelingen (accessed June 2016).

58. Vereecken C, De Henauw S, Maes L et al. (2009) Reliability and validity of a healthy diet determinants questionnaire for adolescents. Public Health Nutr 12, 1830-1838.

59. Gardner B (2012) Habit as automaticity, not frequency. Eur Health Psychol 14, 32-36.

60. Verplanken B \& Orbell S (2003) Reflections on past behavior: a self-report index of habit strength. J Appl Soc Psychol 33, 1313-1330.

61. van Stralen MM, te Velde SJ, Singh AS et al. (2011) EuropeaN energy balance research to prevent excessive weight gain among youth (ENERGY) project: design and methodology of the ENERGY cross-sectional survey. BMC Public Health 11, 65.

62. Roelants M, Hauspie R \& Hoppenbrouwers K (2009) References for growth and pubertal development from birth to 21 years in Flanders, Belgium. Ann Hum Biol 36, 680-694.
63. Cole TJ \& Lobstein T (2012) Extended international (IOTF) body mass index cut-offs for thinness, overweight and obesity. Int J Pediatr Obes 7, 284-294.

64. Muris P, Meesters C, De Kanter E et al. (2005) Behavioural inhibition and behavioural activation system scales for children: relationships with Eysenck's personality traits and psychopathological symptoms. Pers Individ Dif 38, 103-113.

65. Blackman KCA, Zoellner J, Kadir A et al. (2015) Examining the feasibility of smartphone game applications for physical activity promotion in middle school students. Games Health J 4, 409-419.

66. Spook J, Paulussen T, Kok G et al. (2016) Evaluation of a serious self-regulation game intervention for overweightrelated behaviors ('Balance It'): a pilot study. J Med Internet Res 18, 169-182.

67. Partridge SR, Allman-Farinelli M, McGeechan K et al. (2016) Process evaluation of TXT2BFiT: a multi-component mHealth randomised controlled trial to prevent weight gain in young adults. Int J Behav Nutr Phys Act 13, 14.

68. Poels K, de Kort Y \& Ijsselsteijn W (2007) It is always a lot of fun!: exploring dimensions of digital game experience using focus group methodology. In Future Play '07 Proceedings of the 2007 Conference on Future Play, Toronto, Canada, 14-17 November 2007, pp. 83-89. New York: Association for Computing Machinery; available at https://dl.acm.org/ citation.cfm?id=1328202.1328218

69. Bray JW, Schlunder WE, Zarkin GA et al. (2008) Analyzing Data From Nonrandomized Group Studies. Research Triangle Park, NC: RTI Press.

70. Perski O, Blandford A, West R et al. (2017) Conceptualising engagement with digital behaviour change interventions: a systematic review using principles from critical interpretive synthesis. Transl Behav Med 7, 254-267.

71. Garde A, Umedaly A, Abulnaga SM et al. (2015) Assessment of a mobile game ('MobileKids Monster Manor') to promote physical activity among children. Games Health J 4, 149-158.

72. Majumdar D, Koch PA, Lee H et al. (2013) 'Creature-101': a serious game to promote energy balance-related behaviors among middle school adolescents. Games Health J 2, 280-290.

73. Baghaei N, Nandigam D, Casey J et al. (2016) Diabetic Mario: designing and evaluating mobile games for diabetes education. Games Health J 5, 270-278.

74. Crutzen R, Kohl L \& de Vries N (2012) Kennissynthese Online Preventie. Maastricht: Universitaire Pers Maastricht.

75. Stok FM, de Ridder DT, de Vet E et al. (2016) Hungry for an intervention? Adolescents' ratings of acceptability of eatingrelated intervention strategies. BMC Public Health 16, 5.

76. DeSmet A, Van Ryckeghem D, Compernolle S et al. (2014) A meta-analysis of serious digital games for healthy lifestyle promotion. Prev Med 69, 95-107.

77. Cooke LJ, Chambers LC, Anez EV et al. (2011) Facilitating or undermining? The effect of reward on food acceptance. A narrative review. Appetite 57, 493-497.

78. Wiederhold BK (2015) Behavioral health apps abundant, but evidence-based research nearly nonexistent. Cyberpsychol Behav Soc Netw 18, 309-310.

79. Sandholzer M, Deutsch T, Frese T et al. (2015) Predictors of students' self-reported adoption of a smartphone application for medical education in general practice. BMC Med Educ 15, 1.

80. Wardle J, Haase AM, Steptoe A et al. (2004) Gender differences in food choice: the contribution of health beliefs and dieting. Ann Behav Med 27, 107-116.

81. Cooke LJ \& Wardle J (2005) Age and gender differences in children's food preferences. BrJ Nutr 93, 741-746.

82. Kinzie MB \& Joseph DR (2008) Gender differences in game activity preferences of middle school children: implications for educational game design. Educ Technol Res Dev 56, 643-663.

83. Lucas K \& Sherry JL (2004) Sex differences in video game play: a communication-based explanation. Commun Res 31, 499-523. 
84. Scarborough P, Boxer A, Rayner M et al. (2007) Testing nutrient profile models using data from a survey of nutrition professionals. Public Health Nutr 10, 337-345.

85. Scarborough P, Payne C, Agu CG et al. (2013) How important is the choice of the nutrient profile model used to regulate broadcast advertising of foods to children? A comparison using a targeted data set. Eur J Clin Nutr 67 , 815-820.
86. Hedges LV (2007) Effect sizes in cluster-randomized designs. J Educ Behav Stat 32, 341-370.

87. Feingold A (2013) A regression framework for effect size assessments in longitudinal modeling of group differences. Rev Gen Psychol 17, 111-121.

88. Selya AS, Rose JS, Dierker LC et al. (2012) A practical guide to calculating Cohen's $f^{2}$, a measure of local effect size, from PROC MIXED. Front Psychol 3, 111. 